\title{
Vaccine Enthusiasm and Hesitancy in Cancer Patients and the Impact of a Webinar
}

\author{
Amar H. Kelkar ${ }^{1}{ }^{(\mathbb{D}}$, Jodian A. Blake ${ }^{2}$, Kartikeya Cherabuddi ${ }^{3}{ }^{(0)}$, Hailee Cornett ${ }^{4}$, Bobbie L. McKee ${ }^{5}$ \\ and Christopher R. Cogle ${ }^{1, *(D)}$
}

1 Division of Hematology and Oncology, Department of Medicine, College of Medicine, University of Florida, Gainesville, FL 32610, USA; amarhkelkar@gmail.com

2 Office of Community Outreach, Engagement \& Equity, UF Health Cancer Center, Gainesville, FL 32610, USA; blake.j@ufl.edu

3 Division of Infectious Disease \& Global Medicine, Department of Medicine, College of Medicine, University of Florida, Gainesville, FL 32610, USA; kartikeya.cherabuddi@medicine.ufl.edu

4 North Central Florida Cancer Control Collaborative Consortium, Well Florida Council, Gainesville, FL 32606, USA; hcornett@wellflorida.org

5 Florida Cancer Control and Research Advisory Council, H. Lee Moffitt Cancer Center, Tampa, FL 33612, USA; bobbie.mckee@moffitt.org

* Correspondence: Christopher.Cogle@medicine.ufl.edu; Tel.: +1-(352)-273-7493

check for updates

Citation: Kelkar, A.H.; Blake, J.A.; Cherabuddi, K.; Cornett, H.; McKee, B.L.; Cogle, C.R. Vaccine Enthusiasm and Hesitancy in Cancer Patients and the Impact of a Webinar. Healthcare 2021, 9, 351. https://doi.org/ $10.3390 /$ healthcare 9030351

Academic Editor: Pedram Sendi

Received: 11 March 2021

Accepted: 17 March 2021

Published: 19 March 2021

Publisher's Note: MDPI stays neutral with regard to jurisdictional claims in published maps and institutional affiliations.

Copyright: (c) 2021 by the authors. Licensee MDPI, Basel, Switzerland. This article is an open access article distributed under the terms and conditions of the Creative Commons Attribution (CC BY) license (https:// creativecommons.org/licenses/by/ $4.0 /)$.

\begin{abstract}
Background: Vaccine hesitancy and rejection are major threats to controlling coronavirus disease 2019 (COVID-19). There is a paucity of information about the attitudes of cancer patients towards vaccinations and the role of clinical oncologists in influencing vaccination acceptance. (2) Methods: Cancer patients and caregivers were invited to participate in a webinar and two surveys (pre- and post-webinar) assessing intention and thought processes associated with receiving COVID-19 vaccines. (3) Results: Two hundred and sixty-four participants participated in the webinar and registered to take at least one survey. Participants reported receiving most of their COVID-19 vaccine information from their doctor, clinic, or hospital. Before the webinar, $71 \%$ of participants reported the intention to receive a COVID-19 vaccine, $24 \%$ were unsure, and $5 \%$ had no intention of receiving a vaccine. The strongest predictors of vaccine enthusiasm were (a) planning to encourage the vaccination of family, friends, co-workers, and community, and (b) physician recommendation. The chief reason for vaccine hesitancy was a fear of side effects. After the webinar, $82.5 \%$ reported the intention to receive a vaccine, $15.4 \%$ were still unsure, and $2 \%$ stated that they had no intention of receiving a vaccine. The webinar shifted the attitude towards vaccine enthusiasm, despite an already vaccine-enthusiastic population. Communicating about vaccines using positive framing is associated with greater vaccine enthusiasm. (4) Conclusions: Patient education programs co-hosted by multiple stakeholders and delivered by oncologists can increase cancer patient enthusiasm for COVID-19 vaccination.
\end{abstract}

Keywords: COVID-19; SARS-CoV2; vaccine hesitancy; vaccine; public health; patient education

\section{Introduction}

In the historic process of rapidly developing, testing, and approving coronavirus disease 2019 (COVID-19) vaccines for emergency use authorization, certain populations have yet to be fully studied, including cancer patients [1,2]. However, the pressing threat of COVID-19 in cancer patients has supported a broad recommendation for cancer patients to receive vaccinations against COVID-19, with some specific considerations [3-5]. In deploying COVID-19 vaccines, the medical community and government agencies are confronting vaccine hesitancy and the potential for slow vaccine uptake [6-8].

Prior to COVID-19, vaccine hesitancy was a mounting problem in the US and worldwide. In the early months of COVID-19, there were signs of vaccine hesitancy worsening. 
As few as $50 \%$ of people surveyed reported the intention to receive a vaccine, which were still in testing at that point [7,9]. However, more recent surveys have shown declining vaccine hesitancy $[10,11]$. This change in sentiment may have been due to the combination of winter peaks in COVID-19 cases and case fatalities, as well as tangible vaccine emergency authorization. Nonetheless, there remain significant concerns that society will not reach adequate vaccination levels to achieve community immunity [12].

Although vaccine decision-making has been studied among parents, the general public, and health care professionals, there are no studies of vaccine decision-making in cancer patients. Health care professional knowledge and attitudes towards vaccines are known to be important factors in the rate of influenza vaccine uptake [13-15]. This has been further reinforced in recent surveys pertaining to COVID-19 vaccines $[9,11]$. Therefore, as oncologists and cancer patient advocates, we created and presented a webinar on COVID-19 vaccines for cancer patients and caregivers. We hypothesized that the webinar would increase knowledge about vaccinations against COVID-19 and change attitudes from vaccine rejection or hesitancy to vaccine acceptance or enthusiasm.

\section{Materials and Methods}

\subsection{Study Design and Participants}

Through the cooperation of the UF Health Cancer Center, the North Central Florida Regional Cancer Control Collaborative, and the Florida Cancer Control and Research Advisory Council (historically referred to as CCRAB), an educational webinar entitled "Cancer in the Time of Coronavirus: COVID-19 Vaccine" was developed using peerreviewed literature, as well as public health information from the U.S. Centers for Disease Control and Prevention (CDC), U.S. Food and Drug Administration (FDA), World Health Organization (WHO), and New York Times. The webinar was advertised to members of the three co-hosting organizations via emails and social media posts (i.e., Facebook, Twitter, and Instagram). Webinar participation required pre-registration.

This study was approved by the University of Florida Institutional Review Board (IRB-02) for the use of a pre-webinar survey (Figure S1) and post-webinar survey assessing participant demographics, beliefs, and perspectives on vaccines and COVID-19. The prewebinar survey was open from 31 December 2020 to the webinar date, which was 8 January 2021. The post-webinar survey was open from 8 January 2021 to 16 January 2021. Questions regarding vaccine beliefs and plans were designed based on the proposed causes of vaccine hesitancy and prior survey questions presented by the WHO and other studies $[6,16,17]$.

\subsection{Statistical Methods}

The primary aims of the study were to observe endemic vaccine beliefs in a population of cancer patients and caregivers and determine which of these findings might be modifiable with community outreach and engagement. Secondary aims included determining the impact of age, gender, race, active treatment, political affiliation, and other key demographics on beliefs and vaccine plans. All statistical analyses were performed using IBM SPSS Statistics (Armonk, NY, USA). Descriptive statistics such as frequencies, means, and medians were used to analyze participant characteristics, sources of information, stated reasons for vaccine enthusiasm or hesitancy, and intentions to receive a vaccine. Automatic linear modeling for regression was used to create a predictive model of characteristics and beliefs associated with the choice to receive a COVID-19 vaccination. Ensemble modeling was used to boost the ability to identify predictors for vaccine enthusiasm or hesitancy. Paired $t$-tests were employed to compare pre- and post-webinar survey responses from the same individuals and $p$ values $<0.05$ were considered statistically significant.

\section{Results}

\subsection{Participants and Characteristics}

In total, 264 people participated in the webinar and registered to take at least one survey (Figure 1). Of these, 205 (78\%) completed the pre-webinar survey, $138(52 \%)$ 
completed the post-webinar survey, $105(40 \%)$ people completed both the pre-webinar and post-webinar surveys, and $26(10 \%)$ completed neither survey.

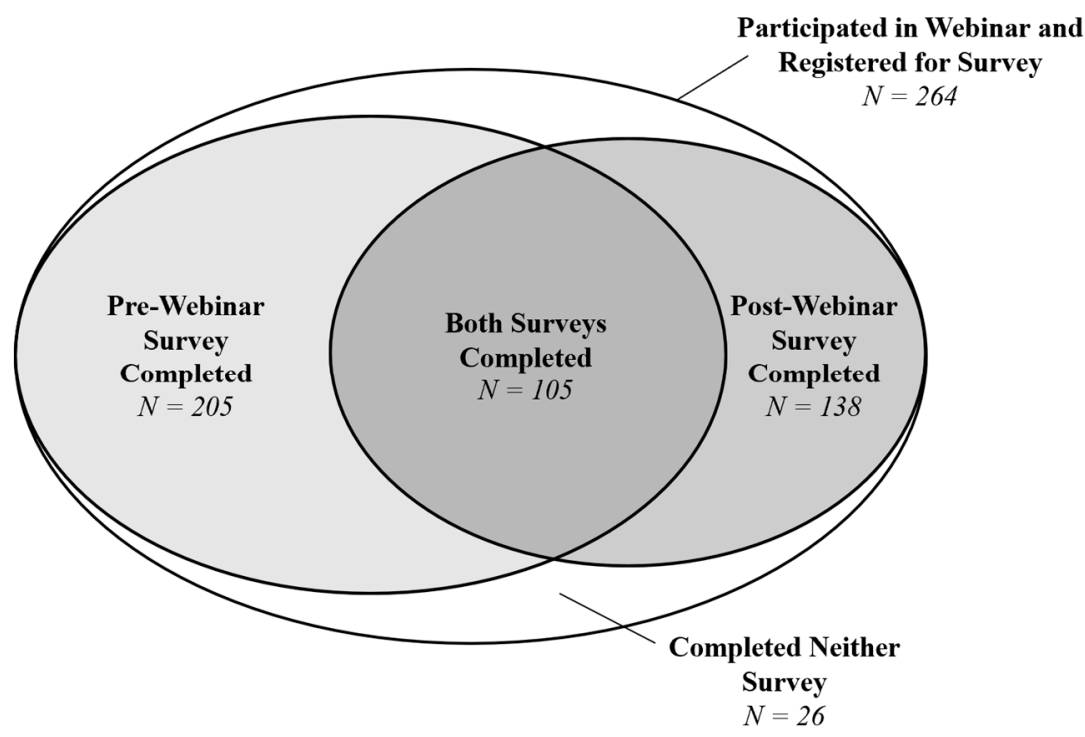

Figure 1. Number of participants who completed the pre-webinar and/or post-webinar surveys.

The characteristics of the three cohorts who completed the pre-webinar, post-webinar, or both surveys are described in Table 1. The characteristics among the cohorts were broadly similar; however, there was a higher percentage of people in the post-webinar cohort who preferred not to answer demographic questions. In general, respondents had personal connections to cancer and were older, White, female, educated, and affluent.

Table 1. Characteristics of webinar participants who completed surveys. Three overlapping cohorts of people (238 unique individuals) completed surveys online before or after participating in a webinar on vaccines and coronavirus. A cohort of 205 people completed the pre-webinar survey, 105 people completed both surveys, and a cohort of 138 people completed the post-webinar survey. The characteristics of race, health insurance, and connections to cancer add up to more than $100 \%$ because respondents were allowed to choose more than one answer. For other characteristics, the total may not equal $100 \%$ because of rounding.

\begin{tabular}{|c|c|c|c|c|c|c|}
\hline \multirow[b]{2}{*}{ Characteristic } & \multicolumn{2}{|c|}{$\begin{array}{l}\text { Pre-Webinar Survey } \\
\text { Completed }(N=205)\end{array}$} & \multicolumn{2}{|c|}{$\begin{array}{l}\text { Both Surveys Completed } \\
\qquad(N=105)\end{array}$} & \multicolumn{2}{|c|}{$\begin{array}{l}\text { Post-Webinar Survey } \\
\text { Completed }(N=138)\end{array}$} \\
\hline & Number & Percent & Number & Percent & Number & Percent \\
\hline \multicolumn{7}{|l|}{ Age (years) } \\
\hline $18-26$ & 10 & $5 \%$ & 4 & $4 \%$ & 4 & $3 \%$ \\
\hline $27-29$ & 4 & $2 \%$ & 0 & $0 \%$ & 1 & $1 \%$ \\
\hline $30-39$ & 20 & $10 \%$ & 9 & $9 \%$ & 9 & $7 \%$ \\
\hline $40-49$ & 32 & $6 \%$ & 14 & $13 \%$ & 15 & $11 \%$ \\
\hline $50-59$ & 35 & $17 \%$ & 15 & $14 \%$ & 15 & $11 \%$ \\
\hline $60-64$ & 33 & $16 \%$ & 20 & $19 \%$ & 25 & $18 \%$ \\
\hline $65-69$ & 19 & $9 \%$ & 11 & $11 \%$ & 1 & $1 \%$ \\
\hline $70-79$ & 39 & $19 \%$ & 23 & $22 \%$ & 25 & $18 \%$ \\
\hline 80 years or older & 11 & $5 \%$ & 9 & $9 \%$ & 11 & $8 \%$ \\
\hline Prefer not to answer & 2 & $1 \%$ & 0 & $0 \%$ & 32 & $23 \%$ \\
\hline
\end{tabular}


Table 1. Cont.

\begin{tabular}{|c|c|c|c|c|c|c|}
\hline \multirow[b]{2}{*}{ Characteristic } & \multicolumn{2}{|c|}{$\begin{array}{l}\text { Pre-Webinar Survey } \\
\text { Completed }(N=205)\end{array}$} & \multicolumn{2}{|c|}{$\begin{array}{l}\text { Both Surveys Completed } \\
\qquad(N=105)\end{array}$} & \multicolumn{2}{|c|}{$\begin{array}{l}\text { Post-Webinar Survey } \\
\text { Completed }(N=138)\end{array}$} \\
\hline & Number & Percent & Number & Percent & Number & Percent \\
\hline \multicolumn{7}{|l|}{ Gender Identity } \\
\hline Man & 40 & $20 \%$ & 25 & $24 \%$ & 27 & $20 \%$ \\
\hline Woman & 161 & $79 \%$ & 78 & $74 \%$ & 88 & $57 \%$ \\
\hline $\begin{array}{l}\text { Nonbinary, genderqueer, } \\
\text { or genderfluid }\end{array}$ & 1 & $0.5 \%$ & 1 & $1 \%$ & 0 & $0 \%$ \\
\hline Prefer not to answer & 3 & $2 \%$ & 1 & $1 \%$ & 23 & $17 \%$ \\
\hline \multicolumn{7}{|l|}{ Sexual Orientation } \\
\hline Heterosexual or "straight" & 191 & $93 \%$ & 97 & $92 \%$ & 108 & $78 \%$ \\
\hline $\begin{array}{l}\text { Homosexual, gay, or } \\
\text { lesbian }\end{array}$ & 3 & $1.5 \%$ & 2 & $2 \%$ & 3 & $2 \%$ \\
\hline Bisexual & 2 & $1 \%$ & 0 & $0 \%$ & 1 & $1 \%$ \\
\hline Other & 1 & $0.5 \%$ & 1 & $1 \%$ & 1 & $1 \%$ \\
\hline Prefer not to answer & 8 & $4 \%$ & 4 & $4 \%$ & 25 & $18 \%$ \\
\hline \multicolumn{7}{|l|}{ Race } \\
\hline $\begin{array}{l}\text { American Indian or Alaska } \\
\text { Native }\end{array}$ & 2 & $1 \%$ & 0 & $0 \%$ & 1 & $1 \%$ \\
\hline Asian or Asian American & 10 & $5 \%$ & 3 & $3 \%$ & 3 & $2 \%$ \\
\hline Black or African American & 12 & $6 \%$ & 7 & $7 \%$ & 8 & $6 \%$ \\
\hline $\begin{array}{l}\text { Native Hawaiian or Other } \\
\text { Pacific Islander }\end{array}$ & 1 & $0.5 \%$ & 1 & $1 \%$ & 1 & $1 \%$ \\
\hline White & 169 & $82 \%$ & 90 & $86 \%$ & 99 & $72 \%$ \\
\hline Other & 7 & $3 \%$ & 2 & $2 \%$ & 2 & 1 \\
\hline Prefer not to answer & 6 & $3 \%$ & 2 & $2 \%$ & 24 & $17 \%$ \\
\hline \multicolumn{7}{|l|}{ Ethnicity } \\
\hline Hispanic or Latinx & 18 & $9 \%$ & 5 & $5 \%$ & 5 & $4 \%$ \\
\hline Not Hispanic or Latinx & 175 & $85 \%$ & 96 & $91 \%$ & 107 & $78 \%$ \\
\hline Prefer not to answer & 12 & $6 \%$ & 4 & $4 \%$ & 26 & $19 \%$ \\
\hline \multicolumn{7}{|l|}{$\begin{array}{l}\text { Highest Level of } \\
\text { Education }\end{array}$} \\
\hline High school or equivalent & 7 & $3 \%$ & 3 & $3 \%$ & 4 & $3 \%$ \\
\hline Some college credits & 15 & $7 \%$ & 7 & $7 \%$ & 9 & $7 \%$ \\
\hline Associate's degree & 15 & $7 \%$ & 7 & $7 \%$ & 9 & $7 \%$ \\
\hline Bachelor's degree & 60 & $29 \%$ & 29 & $28 \%$ & 32 & $23 \%$ \\
\hline $\begin{array}{c}\text { Graduate or professional } \\
\text { degree }\end{array}$ & 103 & $50 \%$ & 56 & $53 \%$ & 60 & $43 \%$ \\
\hline Prefer not to answer & 5 & $2 \%$ & 3 & $3 \%$ & 24 & $17 \%$ \\
\hline
\end{tabular}


Table 1. Cont.

\begin{tabular}{|c|c|c|c|c|c|c|}
\hline \multirow[b]{2}{*}{ Characteristic } & \multicolumn{2}{|c|}{$\begin{array}{l}\text { Pre-Webinar Survey } \\
\text { Completed }(N=205)\end{array}$} & \multicolumn{2}{|c|}{$\begin{array}{l}\text { Both Surveys Completed } \\
\qquad(N=105)\end{array}$} & \multicolumn{2}{|c|}{$\begin{array}{l}\text { Post-Webinar Survey } \\
\text { Completed }(N=138)\end{array}$} \\
\hline & Number & Percent & Number & Percent & Number & Percent \\
\hline \multicolumn{7}{|l|}{ Economics } \\
\hline \multicolumn{7}{|l|}{$\begin{array}{l}\text { Number of people } \\
\text { in household }\end{array}$} \\
\hline 1 & 37 & $18 \%$ & 24 & $23 \%$ & 28 & $20 \%$ \\
\hline 2 & 106 & $52 \%$ & 58 & $55 \%$ & 65 & $47 \%$ \\
\hline 3 & 31 & $15 \%$ & 10 & $10 \%$ & 11 & $8 \%$ \\
\hline 4 & 17 & $8 \%$ & 7 & $7 \%$ & 8 & $6 \%$ \\
\hline 5 & 8 & $4 \%$ & 2 & $2 \%$ & 2 & $1 \%$ \\
\hline 6 & 3 & $1.5 \%$ & 2 & $2 \%$ & 2 & $1 \%$ \\
\hline 7 & 2 & $1 \%$ & 2 & $2 \%$ & 2 & $1 \%$ \\
\hline 8 & 0 & $0 \%$ & 0 & $0 \%$ & 0 & $0 \%$ \\
\hline 9 or more & 1 & $0.5 \%$ & 0 & $0 \%$ & 0 & $0 \%$ \\
\hline Prefer not to answer & 0 & $0 \%$ & 0 & $0 \%$ & 20 & $14 \%$ \\
\hline \multicolumn{7}{|l|}{$\begin{array}{l}\text { Total household income } \\
\text { for } 2020\end{array}$} \\
\hline Less than $\$ 15,000$ & 2 & $1 \%$ & 1 & $1 \%$ & 1 & $1 \%$ \\
\hline$\$ 15,000$ to $\$ 19,999$ & 5 & $2 \%$ & 3 & $3 \%$ & 3 & $2 \%$ \\
\hline$\$ 20,000$ to $\$ 24,999$ & 3 & $1.5 \%$ & 1 & $1 \%$ & 1 & $1 \%$ \\
\hline$\$ 25,000$ to $\$ 34,999$ & 6 & $3 \%$ & 4 & $4 \%$ & 4 & $3 \%$ \\
\hline$\$ 35,000$ to $\$ 49,999$ & 9 & $4 \%$ & 8 & $8 \%$ & 10 & $7 \%$ \\
\hline$\$ 50,000$ to $\$ 74,999$ & 25 & $12 \%$ & 12 & $11 \%$ & 15 & $11 \%$ \\
\hline$\$ 75,000$ to $\$ 99,999$ & 29 & $14 \%$ & 15 & $14 \%$ & 15 & $11 \%$ \\
\hline$\$ 100,000$ and above & 71 & $35 \%$ & 29 & $28 \%$ & 32 & $23 \%$ \\
\hline Prefer not to answer & 55 & $27 \%$ & 32 & $31 \%$ & 57 & $41 \%$ \\
\hline \multicolumn{7}{|l|}{ Health Insurance } \\
\hline Employer offered & 133 & $65 \%$ & 59 & $56 \%$ & 65 & $47 \%$ \\
\hline Private purchase & 37 & $18 \%$ & 21 & $20 \%$ & 26 & $19 \%$ \\
\hline Medicare & 67 & $33 \%$ & 44 & $42 \%$ & 49 & $36 \%$ \\
\hline Veterans Affairs, Tricare & 14 & $7 \%$ & 7 & $7 \%$ & 8 & $6 \%$ \\
\hline Medicaid & 6 & $3 \%$ & 3 & $3 \%$ & 3 & $2 \%$ \\
\hline No health insurance & 1 & $0.5 \%$ & 0 & $0 \%$ & 0 & $0 \%$ \\
\hline Other (student health) & 7 & $3 \%$ & 3 & $3 \%$ & 8 & $6 \%$ \\
\hline Prefer not to answer & 0 & $0 \%$ & 0 & $0 \%$ & 13 & $9 \%$ \\
\hline \multicolumn{7}{|l|}{ Political Affiliation } \\
\hline Democrat & 69 & $34 \%$ & 38 & $36 \%$ & 47 & $34 \%$ \\
\hline Republican & 39 & $19 \%$ & 19 & $18 \%$ & 19 & $14 \%$ \\
\hline Independent & 29 & $14 \%$ & 12 & $11 \%$ & 14 & $10 \%$ \\
\hline Other & 4 & $2 \%$ & 1 & $1 \%$ & 2 & $1 \%$ \\
\hline Prefer not to answer & 64 & $31 \%$ & 35 & $33 \%$ & 56 & $41 \%$ \\
\hline
\end{tabular}


Table 1. Cont.

\begin{tabular}{|c|c|c|c|c|c|c|}
\hline \multirow[b]{2}{*}{ Characteristic } & \multicolumn{2}{|c|}{$\begin{array}{l}\text { Pre-Webinar Survey } \\
\text { Completed }(N=205)\end{array}$} & \multicolumn{2}{|c|}{$\begin{array}{l}\text { Both Surveys Completed } \\
\qquad(N=105)\end{array}$} & \multicolumn{2}{|c|}{$\begin{array}{l}\text { Post-Webinar Survey } \\
\text { Completed }(N=138)\end{array}$} \\
\hline & Number & Percent & Number & Percent & Number & Percent \\
\hline \multicolumn{7}{|l|}{ Connections to Cancer } \\
\hline $\begin{array}{l}\text { Have cancer and actively } \\
\text { receiving treatment }\end{array}$ & 55 & $27 \%$ & 35 & $33 \%$ & 40 & $29 \%$ \\
\hline $\begin{array}{l}\text { Cancer survivor and not } \\
\text { receiving treatment }\end{array}$ & 59 & $29 \%$ & 24 & $24 \%$ & 29 & $17 \%$ \\
\hline $\begin{array}{l}\text { Caregiver to a cancer } \\
\text { patient }\end{array}$ & 20 & $10 \%$ & 12 & $11 \%$ & 12 & $9 \%$ \\
\hline $\begin{array}{c}\text { Friend or family member } \\
\text { to a cancer patient }\end{array}$ & 47 & $23 \%$ & 25 & $24 \%$ & 27 & $20 \%$ \\
\hline Health care provider & 39 & $19 \%$ & 15 & $14 \%$ & 15 & $11 \%$ \\
\hline $\begin{array}{l}\text { Academic researcher or } \\
\text { research staff member }\end{array}$ & 18 & $9 \%$ & 12 & $11 \%$ & 14 & $10 \%$ \\
\hline Government employee & 8 & $4 \%$ & 3 & $3 \%$ & 3 & $2 \%$ \\
\hline $\begin{array}{l}\text { Community-based } \\
\text { organization that serves } \\
\text { people with cancer }\end{array}$ & 20 & $10 \%$ & 10 & $10 \%$ & 10 & $7 \%$ \\
\hline $\begin{array}{l}\text { Health insurance } \\
\text { company employee }\end{array}$ & 1 & $0.5 \%$ & 0 & $0 \%$ & 0 & $0 \%$ \\
\hline No connection to cancer & 2 & $1 \%$ & 0 & $0 \%$ & 0 & $0 \%$ \\
\hline $\begin{array}{l}\text { Other (caregiver to person } \\
\text { with other disease, } \\
\text { for-profit business, family } \\
\text { of health care worker) }\end{array}$ & 12 & $6 \%$ & 6 & $6 \%$ & 8 & $6 \%$ \\
\hline
\end{tabular}

\subsection{Where People Get Information about Vaccines against COVID-19}

Participants reported a variety of sources of information about vaccines against COVID-19 (Figure 2). The most frequently cited source was a doctor, clinic, or hospital. Government agencies such as the CDC and the state Department of Health were the second-ranked source. News outlets were ranked next, followed by family, friends, or coworkers. Participants also reported receiving information from social media, such as Twitter and Facebook, and health insurance companies. Employers were mentioned as other sources of information about vaccines against COVID-19.

\subsection{Characteristics Associated with the Intention to Receive a COVID-19 Vaccine}

Before the webinar, $71 \%$ of people surveyed stated the intention to receive a COVID-19 vaccine, $24 \%$ were unsure, and $5 \%$ had no intention of receiving a vaccine.

The intention to receive a COVID-19 vaccine before the webinar ( $\mathrm{N}=205$ participants) was analyzed in association with 31 variables, including participant demographics and beliefs about vaccines (Figure S1). Automatic linear modeling showed a prediction accuracy of $58 \%$ and identified two strong predictors of vaccine intention, both in the direction of vaccine enthusiasm (positive coefficient) (Table 2). There were no statistically significant predictors of vaccine hesitancy. 


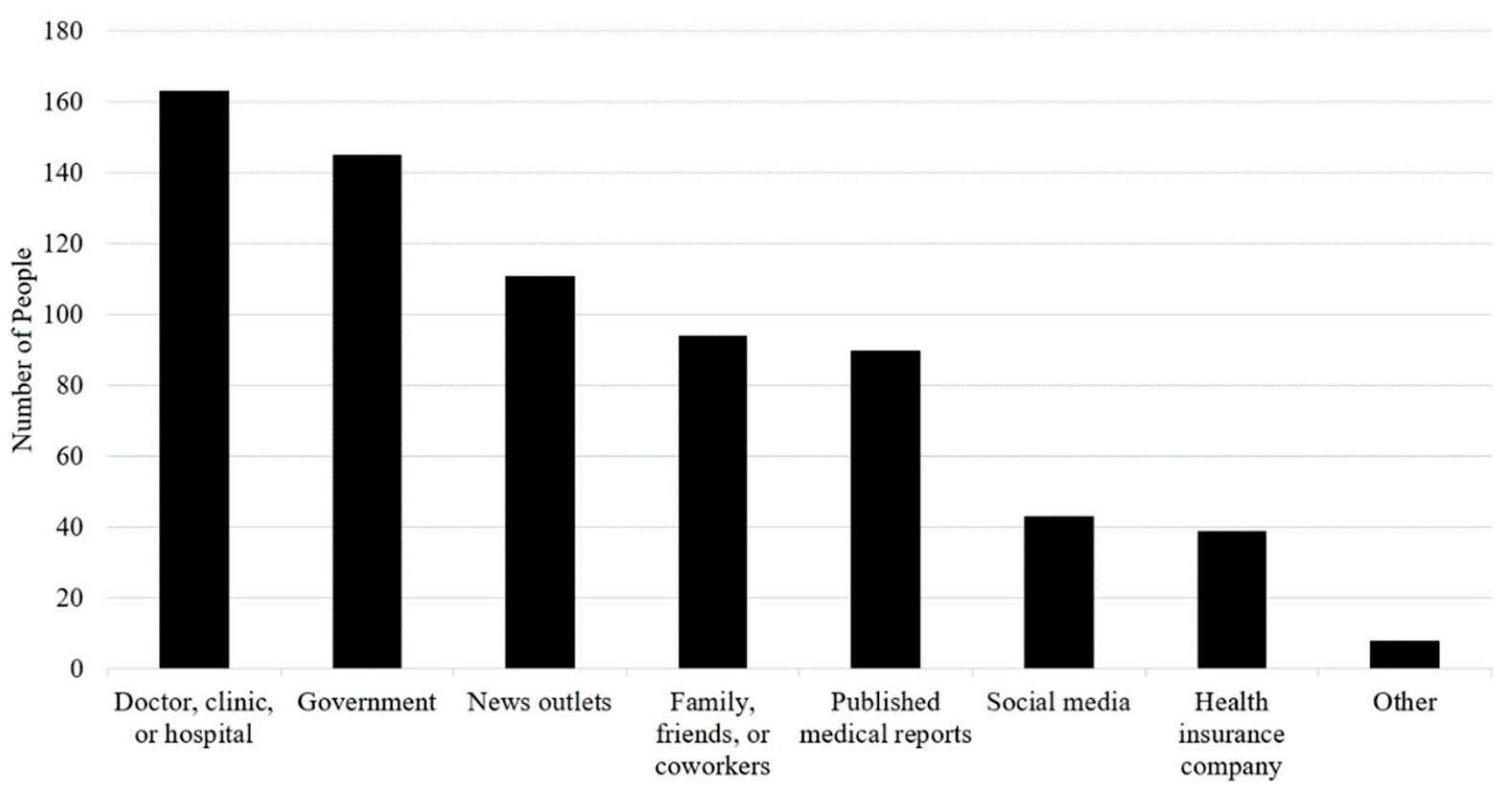

Figure 2. Sources of information about vaccines against coronavirus disease 2019 (COVID-19).

Table 2. Characteristics and beliefs associated with the intention to receive a COVID-19 vaccine. LINEAR modeling of 31 demographic and attitude variables in 205 people prior to participating in a webinar.

\begin{tabular}{cccc}
\hline Characteristic & Coefficient & Significance & Model Importance \\
\hline $\begin{array}{c}\text { I plan to encourage my family, friends, } \\
\text { co-workers, and community to get a } \\
\text { COVID-19 vaccine. }\end{array}$ & 0.260 & $p<0.0001$ & 0.548 \\
\hline $\begin{array}{c}\text { I would take a COVID-19 vaccine if } \\
\text { recommended by my doctor. }\end{array}$ & 0.252 & $p<0.0001$ & 0.351 \\
\hline
\end{tabular}

To boost the model accuracy, ensemble modeling was used for the same 31 demographic and belief variables from 205 participants. This iterative technique achieved a prediction accuracy of $59 \%$ and identified four variables with a higher predictive importance (Figure 3). The top two predictors were the same as predicted by the first linear regression model in Table 2, which confirmed their importance. The boosted model also identified beliefs in vaccine effectiveness and safety as important features associated with the intention to receive a COVID-19 vaccine.

\subsection{Webinar Impact on the Intention to Receive a COVID-19 Vaccine}

Reasons for vaccine hesitancy included concern about side effects from the vaccine $(30 \%)$, a lack of information on COVID-19 vaccine effectiveness (14\%), a lack of trust that the vaccine is safe for cancer patients $(8 \%)$, a lack of information on where or how to get a COVID-19 vaccine (5\%), a fear of needles (4\%), a fear of contracting COVID-19 from the vaccine $(2 \%)$, concern about payment for the vaccine $(2 \%)$, disbelief that the COVID-19 vaccines are effective (1\%), disbelief that the COVID-19 pandemic is significant $(1 \%)$, and an allergy to other vaccines $(1 \%)$. Less frequent reasons for vaccine hesitancy included concerns about vaccine interference with treatment for cancer, interference with fertility or pregnancy, preference to prevent with distancing and masking rather than vaccination, vaccine dilution because of a limited supply, a lack of long-term studies demonstrating safety, distrust of medical research because of historical racial discrimination, and an unsubstantiated claim by one participant that vaccines were made using cells from aborted fetuses. 


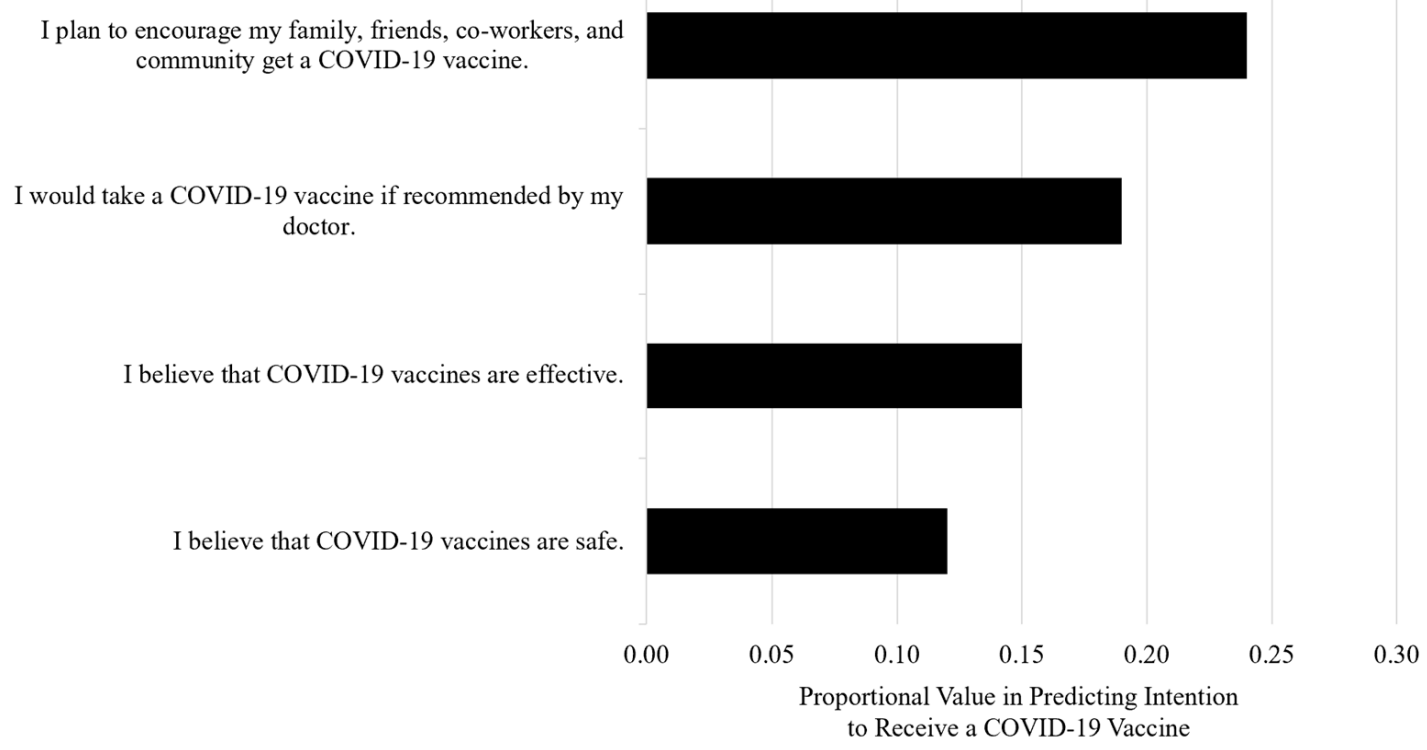

Figure 3. Predictors of the intention to receive a COVID-19 vaccine. Levels of model importance in predicting the intention to receive a COVID-19 vaccine based on webinar participant demographics and beliefs.

To measure the extent by which the webinar changed the intention to receive a COVID-19 vaccine, responses from 97/105 individuals who answered the specific question about vaccine intention in both the pre- and post-webinar survey were studied. After the webinar, 80/97 (82.5\%) of participants stated the intention to receive a COVID-19 vaccine, $15 / 97(15.5 \%)$ were still unsure, and $2(2.1 \%)$ stated no intention to receive a vaccine. The webinar increased the number of 'yes' respondents by 3, decreased the number of 'maybe' respondents by 2 , and decreased the number of 'no' respondents by 1 . Overall, the webinar shifted an already vaccine-enthusiastic population toward even greater vaccine enthusiasm and decreased vaccine hesitancy and rejection.

\subsection{Changes in Beliefs and Perspectives on Vaccines against COVID-19}

Participants were queried before and after the webinar about their beliefs and perspectives on vaccines against COVID-19. In general, participants had favorable views of the vaccines (Figure 4). Despite high baseline agreeableness in relation to vaccines, the webinar further increased participants' positive views of vaccines against COVID-19, specifically increasing agreeance with belief statements about vaccine effectiveness; vaccine safety; vaccine acceptance if recommended by a doctor; extra effort to receive a vaccine; and encouraging their family, friends, coworkers, and community to receive a vaccine.

\subsection{Participants' Understanding of the Vaccine Depends on Communication Framing}

When communicating the risks and benefits of vaccines, or any treatment, physicians must communicate probabilistic information. Clinicians can use different methods to communicate risks. In this study, three communication methods were tested to assess the association with vaccine enthusiasm or hesitancy. Specifically, participants were asked whether they would be willing to receive a vaccine with a $90 \%$ effective rate (positive framing), with a $10 \%$ failure rate (negative framing), or preventing 9 out of 10 people from being infected (frequency). The three answers are equivalent. However, more respondents $(78 \%)$ chose to receive a vaccine worded with positive framing compared to a vaccine worded with frequency $(64 \%)$ or negative framing (56\%) (Figure 5). 


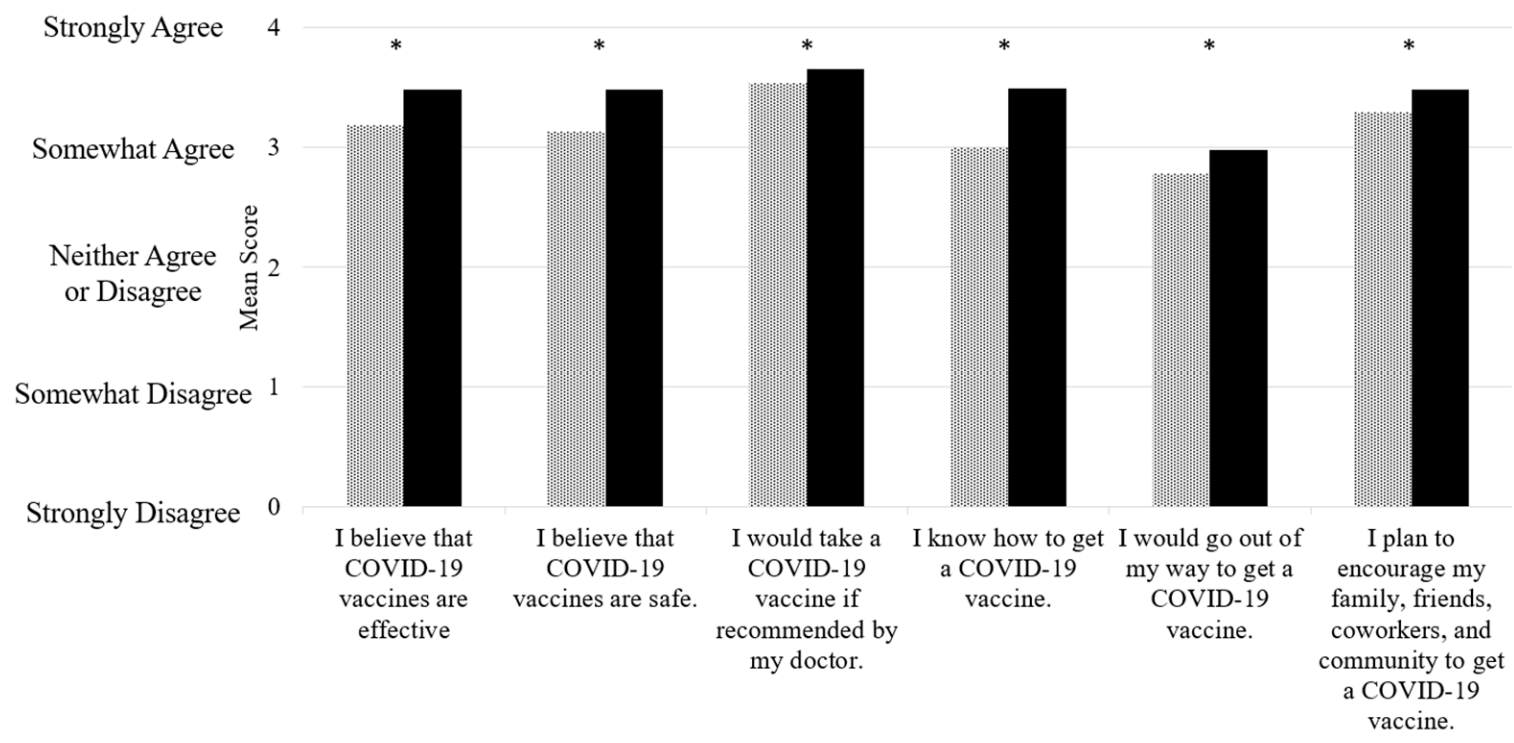

Figure 4. Changes in beliefs and perspectives on vaccines against COVID-19 before and after a webinar. Dotted bars represent data collected before the webinar. Black bars represent data collected after the webinars. A paired $t$-test used to compare before and after scores. ${ }^{*}, p<0.05$.

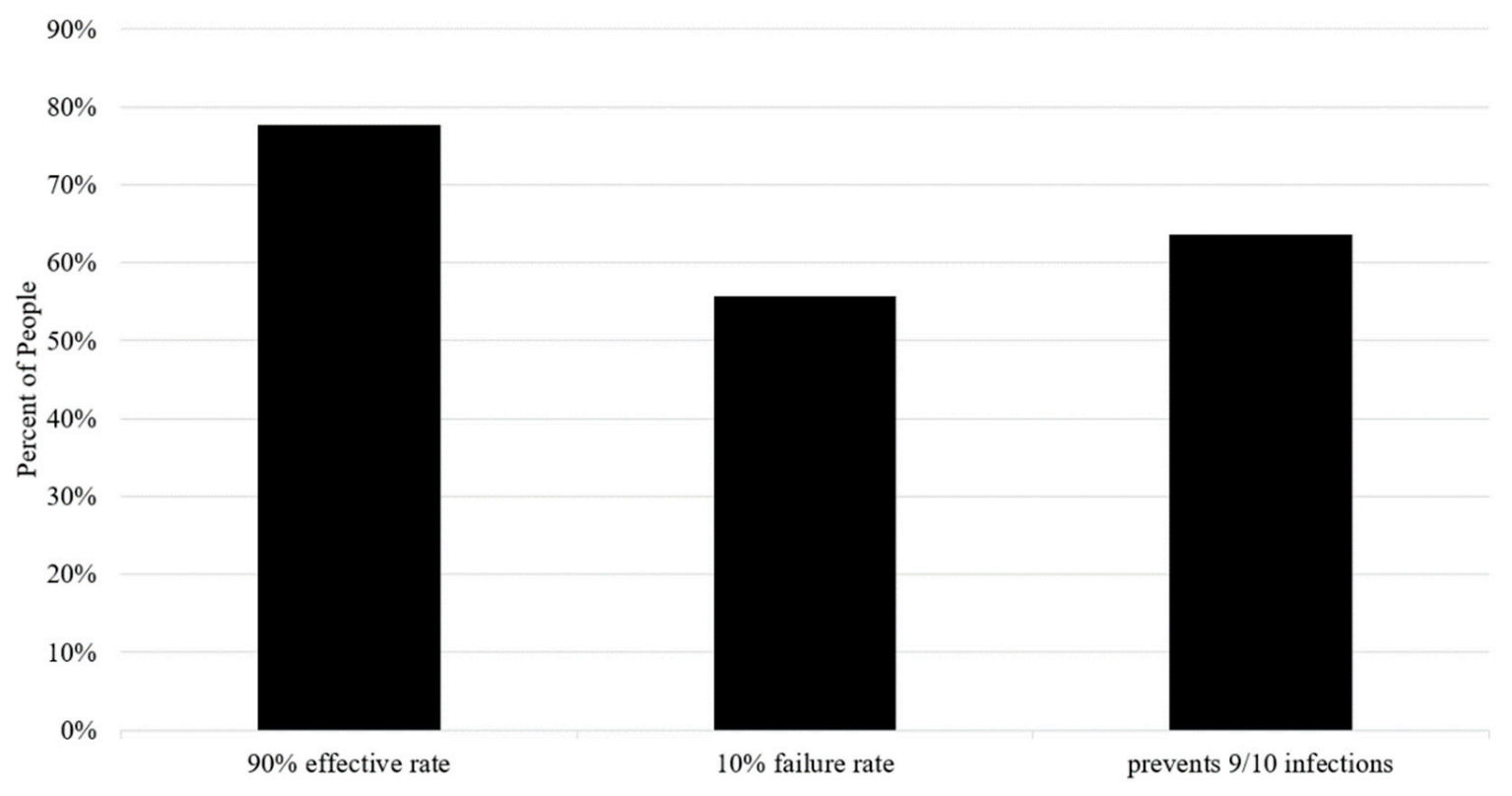

Figure 5. Which vaccine would you take?

\section{Discussion}

This study sheds light on vaccine acceptance among people with a history of cancer and their caregivers, which is a pressing issue in cancer centers and clinics worldwide. With limited time and resources, oncology clinicians, primary care providers, and patient advocacy organizations must choose how best to encourage COVID-19 vaccination for their patients with a history of cancer. Through the use of a webinar and surveys, this study exposed several mechanisms of vaccine hesitancy, but underscores two major points for physicians and patient advocates (Figure 6). First, physician authority is respected for COVID-19 vaccination. This factor was highly associated with positive attitudes toward receiving a COVID-19 vaccine. This finding has been previously reported for other vaccines, such as vaccines against influenza [13-15]. To shift public attitude toward vaccine acceptance, there need to be opportunities for physicians to directly and clearly engage with 
their patients and the public regarding vaccine safety and efficacy. Second, empathy gives purpose to COVID-19 vaccination. Study participants who were concerned about their family, friends, and coworkers were more likely to accept a vaccine for themselves. This worldview of human connection and shared sensibility speaks to the paradox of COVID19 as both predator and prey to community action. Although messaging for COVID-19 vaccination has not yet incorporated empathy, the results from this study suggest that it should. One example might be "vaccinate if not for yourself, then for others."

\section{Vaccine Hesitancy}

\section{Vaccine Enthusiasm}

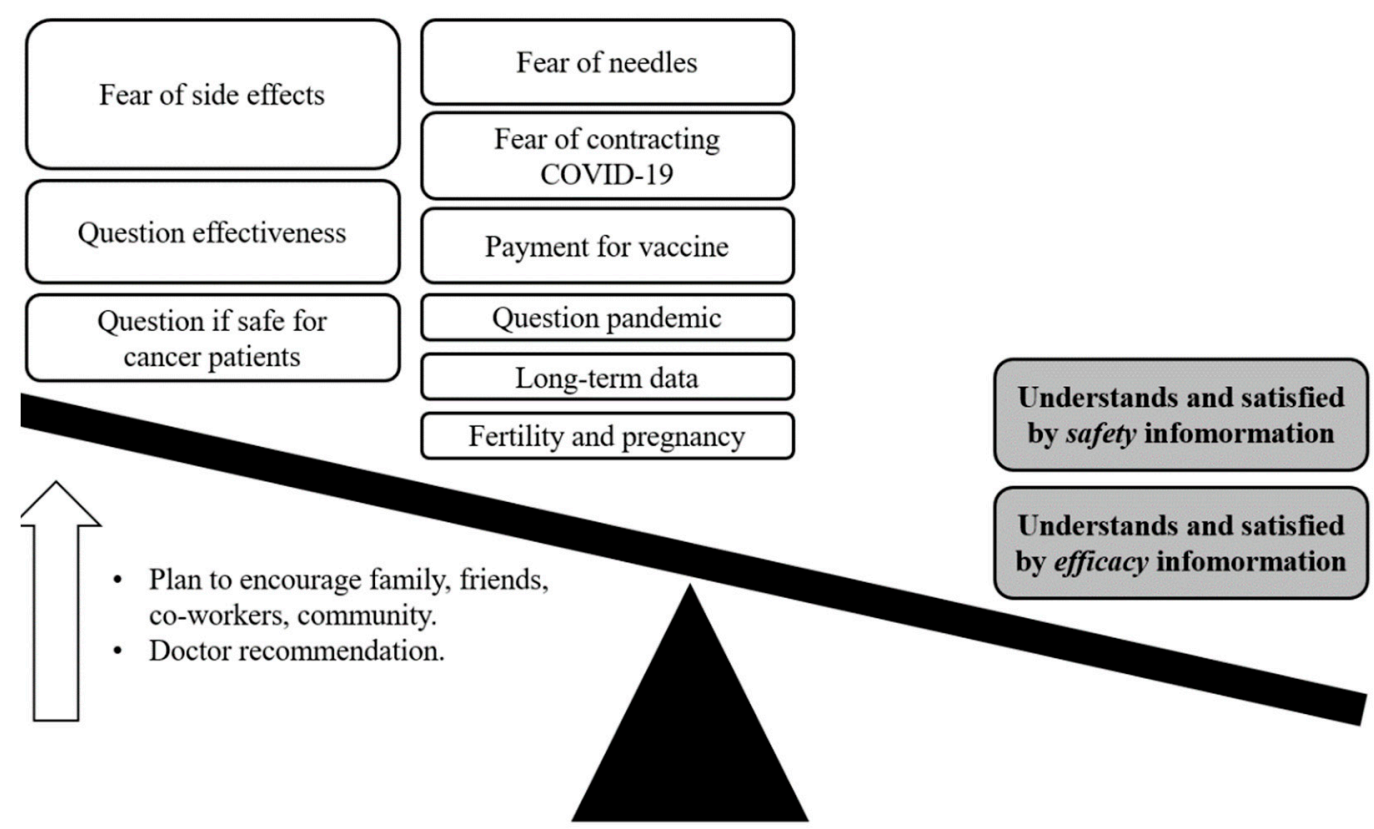

Figure 6. Model of vaccine hesitancy or enthusiasm in cancer patients. The size of the boxes is proportionate to their frequency in the cancer population participating in a webinar on vaccines and COVID-19. The gray boxes indicate the association between factors and the plan to receive a vaccine. The factors next to the up-arrow are associated with decreasing vaccine hesitancy in the cancer population participating in a webinar.

A further evaluation of patient attitudes found that vaccine enthusiasm was boosted by positive impressions of safety and efficacy data of the COVID-19 vaccines. In contrast, a fear of side effects was the chief concern of vaccine-hesitant participants. This study confirms and extends prior reports showing that the most formidable impediments to vaccine acceptance are safety concerns related to adverse effects and that allaying such concerns directly supports vaccine uptake $[6,7,9,14,18,19]$. This places an emphasis on high quality health communications and combating sources of misinformation. While our surveys were not designed to directly identify the role of misinformation in patient beliefs towards vaccines, some of these effects could be seen indirectly. The impact of social group influences is considered a major component of misinformation campaigns, through word of mouth and social media [20,21]. While seen to a lesser extent in our study, both these sources of information were used by many of our webinar attendees. We believe that these sources can be co-opted for the dissemination of high-quality information to promote safe vaccine usage; however, they remain threats in the campaign for vaccine acceptance [20]. This study supports the effort to create and deploy public-facing programs such as our webinar that serve to educate cancer patients about vaccinations [22,23].

This study also found that risk communication impacts attitudes toward COVID-19 vaccination. A higher number of participants elected to receive a vaccine when positive framing was used, compared to frequency wording or negative framing. Similar observations were observed when oncologists described the risks and benefits of chemotherapy 
for cancer [24]. This communication framing effect appears to hold for vaccines. When considering the relatively high educational status of our study participants, these findings raise concern for public susceptibility to negative framing by misinformed or nefarious agents or groups.

A few limitations should be considered when interpreting this study. First, the study population was predominately White, older, female, educated, and affluent, and had both access to and familiarity with videoconferencing and survey responses via the Internet. Of these factors, a White race, older age, higher educational attainment, and higher household income may have predisposed our study population to have a higher baseline level of vaccine enthusiasm $[6,7,9]$. At the baseline, this population expressed vaccine enthusiasm. Furthermore, some participants did not respond to demographic questions, which limited analyses. In the future, a longitudinal study design capturing the impact of the webinar on physically receiving a vaccine would also add value to the study results. Despite these limitations, the webinar still appeared to shift attitudes toward vaccine enthusiasm and away from vaccine rejection.

\section{Conclusions}

This study supports the use of cancer patient education programs about vaccinations where physicians clearly and directly present probabilistic data using positive framing and empathy. These results support the findings of prior studies exploring tools to combat vaccine hesitancy through community-level education. This effort also highlights the importance and opportunity for academic cancer centers, regional cancer collaboratives supported by the CDC and state departments of health, and state cancer councils to synergistically work together for cancer patient advocacy.

Supplementary Materials: The following are available online at https:/ / www.mdpi.com/2227-903 2/9/3/351/s1: Figure S1: Pre-webinar survey.

Author Contributions: Conceptualization, A.H.K. and C.R.C.; methodology, A.H.K. and C.R.C.; software, C.R.C.; validation, C.R.C.; formal analysis, C.R.C.; investigation, A.H.K. and C.R.C.; resources, A.H.K., J.A.B., and C.R.C.; data curation, C.R.C.; writing-original draft preparation, A.H.K. and C.R.C.; writing-review and editing, A.H.K., J.A.B., K.C., H.C., B.L.M., and C.R.C.; visualization, A.H.K. and C.R.C.; supervision, A.H.K., J.A.B., K.C., H.C., B.L.M., and C.R.C.; project administration, A.H.K., J.A.B., K.C., H.C., B.L.M., and C.R.C.; funding acquisition, none. All authors have read and agreed to the published version of the manuscript.

Funding: This research received no external funding. C.R.C. is a National Academy of Medicine Emerging Leader in Health and Medicine.

Institutional Review Board Statement: The study was conducted according to the guidelines of the Declaration of Helsinki, and approved by the University of Florida Institutional Review Board (IRB-2).

Informed Consent Statement: Informed consent was obtained from all subjects involved in the study.

Data Availability Statement: The data presented in this study are available on request from the corresponding author. The data are not publicly available due to participant privacy.

Conflicts of Interest: The authors declare no conflict of interest. The funders had no role in the design of the study; in the collection, analyses, or interpretation of data; in the writing of the manuscript; or in the decision to publish the results.

\section{References}

1. Polack, F.P.; Thomas, S.J.; Kitchin, N.; Absalon, J.; Gurtman, A.; Lockhart, S.; Perez, J.L.; Pérez Marc, G.; Moreira, E.D.; Zerbini, C.; et al. Safety and Efficacy of the BNT162b2 mRNA Covid-19 Vaccine. N. Engl. J. Med. 2020, 383, 2603-2615. [CrossRef] [PubMed]

2. Baden, L.R.; El Sahly, H.M.; Essink, B.; Kotloff, K.; Frey, S.; Novak, R.; Diemert, D.; Spector, S.A.; Rouphael, N.; Creech, C.B.; et al. Efficacy and Safety of the mRNA-1273 SARS-CoV-2 Vaccine. N. Engl. J. Med. 2021, 384, 403-416. [CrossRef] [PubMed] 
3. American Society of Hematology \& American Society for Transplantation and Cellular Therapy. ASH-ASTCT COVID-19 and Vaccines: Frequently Asked Questions. Available online: https://www.hematology.org/covid-19/ash-astct-covid-19-andvaccines (accessed on 18 March 2021).

4. American Society of Clinical Oncology. COVID-19 Vaccine \& Patients with Cancer. Available online: https://www.asco.org/ asco-coronavirus-resources / covid-19-vaccines-patients-cancer (accessed on 18 March 2021).

5. National Comprehensive Cancer Network. Cancer and COVID-19 Vaccination (Version 1.0 1/22/2021). Available online: https://www.nccn.org/covid-19/pdf/COVID-19_Vaccination_Guidance_V1.0.pdf (accessed on 18 March 2021).

6. $\quad$ Lazarus, J.V.; Ratzan, S.C.; Palayew, A.; Gostin, L.O.; Larson, H.J.; Rabin, K.; Kimball, S.; El-Mohandes, A. A global survey of potential acceptance of a COVID-19 vaccine. Nat. Med. 2020, 27, 225-228. [CrossRef] [PubMed]

7. Fisher, K.A.; Bloomstone, S.J.; Walder, J.; Crawford, S.; Fouayzi, H.; Mazor, K.M. Attitudes Toward a Potential SARS-CoV-2 Vaccine. Ann. Intern. Med. 2020, 173, 964-973. [CrossRef] [PubMed]

8. Macdonald, N.E. Vaccine hesitancy: Definition, scope and determinants. Vaccine 2015, 33, 4161-4164. [CrossRef]

9. Lin, C.; Tu, P.; Beitsch, L.M. Confidence and Receptivity for COVID-19 Vaccines: A Rapid Systematic Review. Vaccines 2020, 9, 16. [CrossRef] [PubMed]

10. Attwell, K.; Betsch, C.; Dubé, E.; Sivelä, J.; Gagneur, A.; Suggs, L.S.; Picot, V.; Thomson, A. Increasing vaccine acceptance using evidence-based approaches and policies: Insights from research on behavioural and social determinants presented at the 7 th Annual Vaccine Acceptance Meeting. Int. J. Infect. Dis. 2021. [CrossRef]

11. Press Ganey Associates LLC. Vaccine Hesitancy and Acceptance: Data Segmentation Helps Address Barriers 4 February 2021. Available online: https://www.pressganey.com/resources/white-papers/vaccine-hesitancy-and-acceptance (accessed on 18 March 2021).

12. McNeil, D. How Much Herd Immunity Is Enough? Available online: https://www.nytimes.com/2020/12/24/health/herdimmunity-covid-coronavirus.html (accessed on 18 March 2021).

13. Clark, S.J.; Cowan, A.E.; Wortley, P.M. Influenza vaccination attitudes and practices among US registered nurses. Am. J. Infect. Control 2009, 37, 551-556. [CrossRef] [PubMed]

14. Hollmeyer, H.G.; Hayden, F.; Poland, G.; Buchholz, U. Influenza vaccination of health care workers in hospitals-A review of studies on attitudes and predictors. Vaccine 2009, 27, 3935-3944. [CrossRef] [PubMed]

15. Posfay-Barbe, K.M. How Do Physicians Immunize Their Own Children? Differences Among Pediatricians and Nonpediatricians. Pediatrics 2005, 116, e623-e633. [CrossRef]

16. Dubé, E.; Laberge, C.; Guay, M.; Bramadat, P.; Roy, R.; Bettinger, J.A. Vaccine hesitancy. Hum. Vaccines Immunother. 2013, 9 , 1763-1773. [CrossRef] [PubMed]

17. Larson, H.J.; Jarrett, C.; Schulz, W.S.; Chaudhuri, M.; Zhou, Y.; Dube, E.; Schuster, M.; MacDonald, N.E.; Wilson, R.; Hesitancy, S.W.G.o.V. Measuring vaccine hesitancy: The development of a survey tool. Vaccine 2015, 33, 4165-4175. [CrossRef] [PubMed]

18. Wong, M.C.S.; Wong, E.L.Y.; Huang, J.; Cheung, A.W.L.; Law, K.; Chong, M.K.C.; Ng, R.W.Y.; Lai, C.K.C.; Boon, S.S.; Lau, J.T.F.; et al. Acceptance of the COVID-19 vaccine based on the health belief model: A population-based survey in Hong Kong. Vaccine 2021, 39, 1148-1156. [CrossRef] [PubMed]

19. Kreps, S.; Prasad, S.; Brownstein, J.S.; Hswen, Y.; Garibaldi, B.T.; Zhang, B.; Kriner, D.L. Factors Associated With US Adults' Likelihood of Accepting COVID-19 Vaccination. JAMA Netw. Open 2020, 3, e2025594. [CrossRef]

20. Jarrett, C.; Wilson, R.; O'Leary, M.; Eckersberger, E.; Larson, H.J. Strategies for addressing vaccine hesitancy-A systematic review. Vaccine 2015, 33, 4180-4190. [CrossRef]

21. Wilson, S.L.; Wiysonge, C. Social media and vaccine hesitancy. BMJ Glob. Health 2020, 5, e004206. [CrossRef] [PubMed]

22. Abbasi, J. COVID-19 Conspiracies and Beyond: How Physicians Can Deal with Patients' Misinformation. JAMA 2021, 325, 208. [CrossRef] [PubMed]

23. Dror, A.A.; Eisenbach, N.; Taiber, S.; Morozov, N.G.; Mizrachi, M.; Zigron, A.; Srouji, S.; Sela, E. Vaccine hesitancy: The next challenge in the fight against COVID-19. Eur. J. Epidemiol. 2020, 35, 775-779. [CrossRef] [PubMed]

24. van de Water, L.F.; van Kleef, J.J.; Dijksterhuis, W.P.M.; Henselmans, I.; van den Boorn, H.G.; Vaarzon Morel, N.M.; Schut, K.F.; Daams, J.G.; Smets, E.M.A.; van Laarhoven, H.W.M. Communicating treatment risks and benefits to cancer patients: A systematic review of communication methods. Qual. Life Res. 2020, 29, 1747-1766. [CrossRef] [PubMed] 